\title{
RhC Phenotyping, Adsorption/Elution Test, and SSP-PCR: The Combined Test for D-Elute Phenotype Screening in Thai RhD-Negative Blood Donors
}

\author{
Songsak Srijinda, ${ }^{1,2}$ Chamaiporn Suwanasophon, ${ }^{3}$ \\ Unchalee Visawapoka, ${ }^{4}$ and Malinee Pongsavee ${ }^{5}$ \\ ${ }^{1}$ Graduate Program in Medical Technology, Faculty of Allied Health Sciences, Thammasat University, \\ Rangsit Campus, Rangsit, Patumthani 12121, Thailand \\ ${ }^{2}$ Division of Blood Bank, Department of Pathology, Phramongkutklao Hospital, Bangkok 10400, Thailand \\ ${ }^{3}$ Division of Immunology, Department of Clinical Pathology, Army Institute of Pathology, Bangkok 10400, Thailand \\ ${ }^{4}$ Department of Biochemistry, Phramongkutklao College of Medicine, Bangkok 10400, Thailand \\ ${ }^{5}$ Department of Medical Technology, Faculty of Allied Health Sciences, Thammasat University, \\ Rangsit Campus, Rangsit, Patumthani 12121, Thailand
}

Correspondence should be addressed to Malinee Pongsavee, malineep@tu.ac.th

Received 22 September 2012; Accepted 14 October 2012

Academic Editors: A. Pekrun and A. M. Will

Copyright (c) 2012 Songsak Srijinda et al. This is an open access article distributed under the Creative Commons Attribution License, which permits unrestricted use, distribution, and reproduction in any medium, provided the original work is properly cited.

The Rhesus (Rh) blood group is the most polymorphic human blood group and it is clinically significant in transfusion medicine. Especially, D antigen is the most important and highly immunogenic antigen. Due to anti-D, it is the cause of the hemolytic disease of the newborn and transfusion reaction. About $0.1 \%-0.5 \%$ of Asian people are RhD-negative, whereas in the Thai population, the RhD-negative blood type only occurs in $0.3 \%$. Approximately $10 \%-30 \%$ of RhD-negative in Eastern Asian people actually were D-elute (DEL) phenotype, the very weak D antigen that cannot be detected by indirect antiglobulin test (IAT). There are many reports about anti-D immunization in $\mathrm{RhD}$-negative recipients through the transfusion of red blood cells from individuals with DEL phenotype. D-elute phenotype screening in Thai RhD-negative blood donors was studied to distinguish true RhD-negative from DEL phenotype. A total of 254 Thai serologically RhD-negative blood donors were tested for RhCE phenotypes and anti-D adsorption/elution test. In addition, $\mathrm{RhC}(+)$ samples were tested for RHD 1227A allele by SSP-PCR technique. The RhD-negative phenotype samples consisted of 131 ccee, 4 ccEe, 1 ccEE, 101 Ccee, 16 CCee, and 1 CcEe. The 42 Ccee and 8 CCee phenotype samples were typed as DEL phenotype and 96\% of DEL samples were positive for RHD 1227A allele. The incidence of RhC $(+)$ was $46.4 \%$, and 48 of the $118 \mathrm{RhC}(+)$ samples were positive for both anti-D adsorption/elution test and SSP-PCR technique for RHD 1227A allele. The sensitivity and specificity were $96 \%$ and 100\%, respectively, for RHD 1227A detection as compared with the adsorption/elution test. In conclusion, $\mathrm{RhC}(+)$ phenotype can combine with anti-D adsorption/elution test and RHD 1227A allele SSP-PCR technique for distinguishing true RhD-negative from DEL phenotype.

\section{Introduction}

Rhesus (Rh) antigens are acylated red cell transmembrane protein with a molecular weight of $30-32 \mathrm{kDa}$ and encoded by two highly homologous genes, RHD and RHCE. Both genes are located on chromosome 1p34.3-1p36.1 and are about 30,000 base pairs apart [1]. They have opposite orientation and are highly homologous retaining more than $90 \%$ identity $[2,3]$. The Rh blood group is the most polymorphic human blood group system, of high clinically significance in transfusion medicine. D-antigen is the most important and highly immunogenic antigen. The anti-D alloantibody causes the hemolytic disease of the newborn and transfusion reaction. Persons are clinically classified as $\mathrm{RhD}$-positive or $\mathrm{RhD}$-negative depending on the presence or absence of $\mathrm{D}$ antigen on the red cell surface. The $\mathrm{RhD}$-negative 
trait can be generated by multiple genetic mechanisms, which have been shown to be ethnic group-dependent. In most $\mathrm{RhD}$-negative Caucasoid individuals, an $\mathrm{RhD}$-negative phenotype is associated with the deletion of RHD gene between the upstream and downstream Rhesus boxes and hybrid Rhesus boxes present on both chromosomes $[4,5]$. However, total deletion of RHD gene only accounts for $10 \%-23 \%$ of $\mathrm{RhD}$-negative in African [6] and 60\%-70\% of $\mathrm{RhD}$-negative in Asian populations [7-10]. In addition, about $15 \%$ of Caucasoid people are RhD-negative while in the Asian population, RhD-negative blood type occurs in only $0.1 \%-0.5 \%$ of the population [10-13]. In contrast to RhD-negative Caucasoid persons, approximately 10\%-30\% of Eastern Asian people who are RhD-negative are DEL phenotype, a rare variant of Rh system in which the $\mathrm{D}$ antigen is detectable only by adsorption/elution test $[10,11$, 13-15].

The DEL phenotype is serologically designated as a quantitative variant of $\mathrm{D}$ antigen. A small amount of antiD can be eluted from DEL red blood cells (RBCs) after it is incubated with anti-D, although there is no agglutination by the indirect antiglobulin test (IAT). Most DEL donors are typed as $\mathrm{RhD}$-negative because the routine serological typing does not distinguish RhD-negative from the $\mathrm{DEL}$ phenotype. Previous reports have indicated that the presence of the RHD gene in DEL samples seems to relate strictly to the $\mathrm{RhC}$ phenotype with high incidence of $\mathrm{RhC}(+)$ in the apparent RhD-negative persons [12, 16, 17]. All DEL persons with intact RHD gene showed the CC or Cc phenotype but not the cc phenotype. However, the most frequent cause for DEL phenotype in East Asians is the RHD $1227 \mathrm{~A}$ allele $[7,13,18]$. In addition, the commercial kit for adsorption/elution test is simple and practical for use in all transfusion patients. Three cases of anti-D immunization by DEL red blood cells have been reported in Austria, Japan, and Korea [19-21]. There is no report about DEL phenotype in Thai RhD-negative blood. Therefore, we investigated the combined performance test of RhC phenotyping, anti-D adsorption/elution test, and RHD 1227A specific sequence primer-polymerase chain reaction (SSP-PCR) for distinguish true $\mathrm{RhD}$-negative from DEL phenotype in the 254 Thai RhD-negative blood donors. The combined test for DEL detection may help to ensure safety in blood transfusion and prevent anti-D alloimmunization without adding much to the costs and time consumption and without incurring an avoidable wastage of $\mathrm{RhD}$-negative blood units that are always not available in Thai and other Asian population.

\section{Materials and Methods}

2.1. Blood Samples. A total of $254 \mathrm{RhD}$-negative unrelated blood donors were leftover blood samples which were kindly provided by National Blood Centre, The Thai Red Cross Society. The Thai RhD-negative blood samples were used in this study and the other races were excluded with a donor history record. In addition, the blood samples were preserved in citrate phosphate dextrose (CPD) solution and typed as $\mathrm{RhD}$-negative by routine indirect antiglobulin test. All blood samples were tested for RhCE phenotyping and anti$\mathrm{D}$ adsorption/elution test. The RhD-negative samples with $\mathrm{RhC}(+)$ were further tested for RHD 1227A polymorphism by specific sequence primer-polymerase chain reaction (SSPPCR) technique. This research was approved by Thammasat University Ethics Committee.

2.2. Serological RhCE Phenotyping. The $254 \mathrm{RhD}$-negative blood samples were serotyped for $\mathrm{RhC/c}$ and $\mathrm{RhE} / \mathrm{e}$ antigens by using agglutinating monoclonal anti-C, anti-c, anti-E, and anti-e reagents (DiaClon, DiaMed, Switzerland) in the saline tube test $(20 \mu \mathrm{L}$ of specific monoclonal antibody and $50 \mu \mathrm{L}$ of $3 \%$ red blood cells (RBCs) in saline were added to test tube, centrifuged at 3,400 rpm 15 seconds, and the agglutination was read).

2.3. Adsorption/Elution Test. Adsorption/elution tests were performed on 254 samples. For anti-D adsorption to red blood cells (RBCs), $200 \mu \mathrm{L}$ of RBCs was incubated for 1 hour at $37^{\circ} \mathrm{C}$ with $200 \mu \mathrm{L}$ of monoclonal anti-D IgG (National Blood Centre, The Thai Red Cross Society). The cells were washed and the eluate was prepared by acid elution technique (DiaCidel, DiaMed, Switzerland). The eluates and the last washed supernatants were used for indirect antiglobulin test (IAT) against $\mathrm{RhD}$-positive and $\mathrm{RhD}$-negative cells by using column agglutination technique (LISS/Coombs'card, DiaMed, Switzerland), $25 \mu \mathrm{L}$ of eluate (or last supernatant), and $50 \mu \mathrm{L}$ of $1 \% \mathrm{D}$ positive RBCs in modified LISS (DiluentII, DiaMed, Switzerland) were added to LISS/Coombs'card, incubated at $37^{\circ} \mathrm{C} 15$ minutes, centrifuged at $1,030 \mathrm{rpm}$ for 10 minutes, and the agglutination was read.

2.4. Specific Sequence Primer-Polymerase Chain Reaction (SSP-PCR) Analysis for RHD 1227A Allele. Genomic DNA was extracted by using a commercial kit (AxyPrep Blood Genomic DNA Miniprep Kit, Axygen Biosciences, CA, USA). To screen for the RHD 1227A allele in all samples with $\mathrm{RhC}(+)$, the forward primer for $\mathrm{RHD}$ 1227A allele: $5^{\prime}$-GATGACCAAGTTTTCTGGAAA- ${ }^{\prime}$, and the reverse primer for RHD 1227A: 5'-GTTCTGTCACCCGCATGTCAG-3' were used in amplified a $348 \mathrm{bp}$ product. Another pair of nucleotides (the forward primer: $5^{\prime}$ GCCTTCCCAACCATTCCCTTA- $3^{\prime}$, the reverse primer: $5^{\prime}$ TAGACGTTGCTGTCAGAGGC- $3^{\prime}$ ) were incubated as an internal control to generate a $629 \mathrm{bp}$ PCR fragment from the growth hormone gene. The PCR reactions were performed at a total volume $10 \mu \mathrm{L}$. It contained $1 \mu \mathrm{L}$ of genomic DNA, $0.5 \mathrm{U}$ DNA polymerase, $200 \mu \mathrm{M}$ dNTPs, primers, and $2.5 \mathrm{mM} \mathrm{MgCl}_{2}$ in the buffer provided by the manufacturer. Forty cycles were programmed on the thermocycler (TGradient, Biometra) as follows: denaturation at $94^{\circ} \mathrm{C}$ for 5 minutes, then 35 cycles of 30 seconds at $94^{\circ} \mathrm{C}, 40$ seconds at $68^{\circ} \mathrm{C}$, and 30 seconds at $72^{\circ} \mathrm{C}$. PCR products were visualized in a $2 \%$ agarose gel. 
TABLE 1: The incidence of RhD-negative, DEL, and true D-negative in Thai population.

\begin{tabular}{|c|c|c|c|}
\hline Number of apparent Rh phenotypes & $254 \mathrm{RhD}$-negative & $50 \mathrm{DEL}$ & 204 True D-negative \\
\hline \multicolumn{4}{|l|}{$\mathrm{C}(+)$} \\
\hline CCee & $16(6.3 \%)$ & $8(16.0 \%)$ & $8(3.9 \%)$ \\
\hline CCEe & 0 & 0 & 0 \\
\hline Ccee & $101(39.7 \%)$ & $42(84.0 \%)$ & $59(28.9 \%)$ \\
\hline $\mathrm{CcEe}$ & $1(0.4 \%)$ & 0 & $1(0.5 \%)$ \\
\hline $\mathrm{CcEE}$ & 0 & 0 & 0 \\
\hline Subtotal & $118(46.4 \%)$ & $50(100 \%)$ & $68(33.3 \%)$ \\
\hline \multicolumn{4}{|l|}{$\mathrm{C}(-)$} \\
\hline Ccee & $131(51.6 \%)$ & 0 & $131(64.2 \%)$ \\
\hline ccEe & $4(1.6 \%)$ & 0 & $4(2.0 \%)$ \\
\hline ccEE & $1(0.4 \%)$ & 0 & $1(0.5 \%)$ \\
\hline Subtotal & $136(53.6 \%)$ & 0 & $136(66.7 \%)$ \\
\hline Total & $254(100 \%)$ & $50(100 \%)$ & $204(100 \%)$ \\
\hline
\end{tabular}

\section{Results}

As described in Table 1, the $254 \mathrm{RhD}$-negative samples consisted of 131 ccee, 4 ccEe, 1 ccEE, 101 Ccee, 16 CCee, and 1 CcEe. Among them 19.7\% (50/254) were typed as DEL phenotype by anti-D adsorption/elution test that consisted of 42 Ccee and 8 CCee. The incidence of $\mathrm{RhC}(+)$ of the apparent Thai $\mathrm{RhD}$-negative blood donors was $46.4 \%$ (118/254). In addition, the incidence of DEL phenotype in $\mathrm{RhC}(+)$ persons as determined by adsorption/elution test was $42.4 \%$ (50/118). The distribution of each RhCE phenotype among the $254 \mathrm{RhD}$-negative samples in this study and the previous report from Taiwan, Hong Kong, and Japan were summarized in Table 2. The incidence of RhD-negative and DEL phenotype were similar among Thai, Taiwanese, and Hong Kong populations. The incidence of true D-negative in Thai population was significantly different from Taiwanese and Hong Kong populations $(P<0.05)$. Statistically, it was significantly different as compared with the Japanese population $(P<0.05)$. However, it is of great interest to note that all DEL persons were highly associated with $\mathrm{RhC}(+)$ phenotype in all 4 populations.

RHD 1227A polymorphism was detected by SSP-PCR in $\mathrm{RhC}(+)$ individuals (Figure 1). The comparative results of SSP-PCR for RHD 1227A and adsorption/elution test in the $118 \mathrm{RhC}(+)$ samples were shown in Table 3 . The 48 of the $50 \mathrm{RhD}$ adsorption/elution positive (DEL) samples were also positive for RHD 1227A by SSP-PCR analysis (sensitivity = $96 \%$ ), and all of RhD adsorption/elution negative samples were negative $($ specificity $=100 \%)$. However, only 2 samples revealed an inconsistent result detected by these two methods.

\section{Discussion and Conclusions}

DEL phenotype is the weakest known RhD-positive phenotype in the $\mathrm{Rh}$ blood group system. A normal RhDpositive red blood cell (RBC) has about 10,000-30,000 D sites per cell depending on the Rh genotypes [22], while the number of D sites on DEL RBC is less than 30 per cell [19].

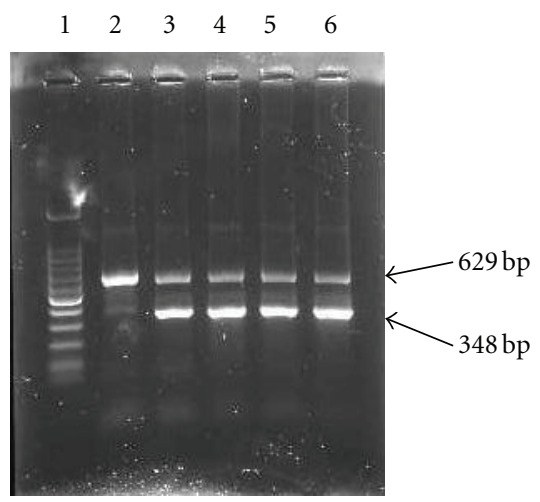

FIGURE 1: Specific sequence primer-polymerase chain reaction (SSP-PCR) analysis of RHD 1227A polymorphism. The products of SSP-PCR after separation on 2\% agarose gel were shown. The specific product were $348 \mathrm{bp}$, and the internal control product, $629 \mathrm{bp}$, based on human growth hormone were amplified. Lane 1 $=100 \mathrm{bp}$ ladder marker, lane 2 = true $\mathrm{RhD}$-negative, and lane 3, 4, 5 , and $6=$ RHD1227A positive.

The incidence of DEL phenotype in Europeans is very low. In contrast, DEL is very common in Eastern Asia such as China, Japan and Korea, and about 10-30\% apparently $\mathrm{RhD}$-negative individuals are DEL phenotypes. However, no data has been collected to indicate the frequency of DEL in Africans.

In this study, approximately $20 \%$ of apparently Thai RhD-negative blood donors were DEL phenotype by anti$\mathrm{D}$ adsorption/elution test. It is in consistence with the other reports; both Ccee and CCee phenotype were highly prevalent in DEL persons of Taiwanese, Japanese, and Hong Kong populations, whereas the prevalence of the CcEe phenotype was about $44 \%$ in DEL group of the Japanese population (Table 2 ). DEL persons were highly associated with the $\mathrm{RhC}(+)$ phenotype in Thai, Taiwanese, Japanese, and Hong Kong populations.

For distinguishing the true RhD-negative from DEL phenotype in clinical laboratory, we found that the presence 


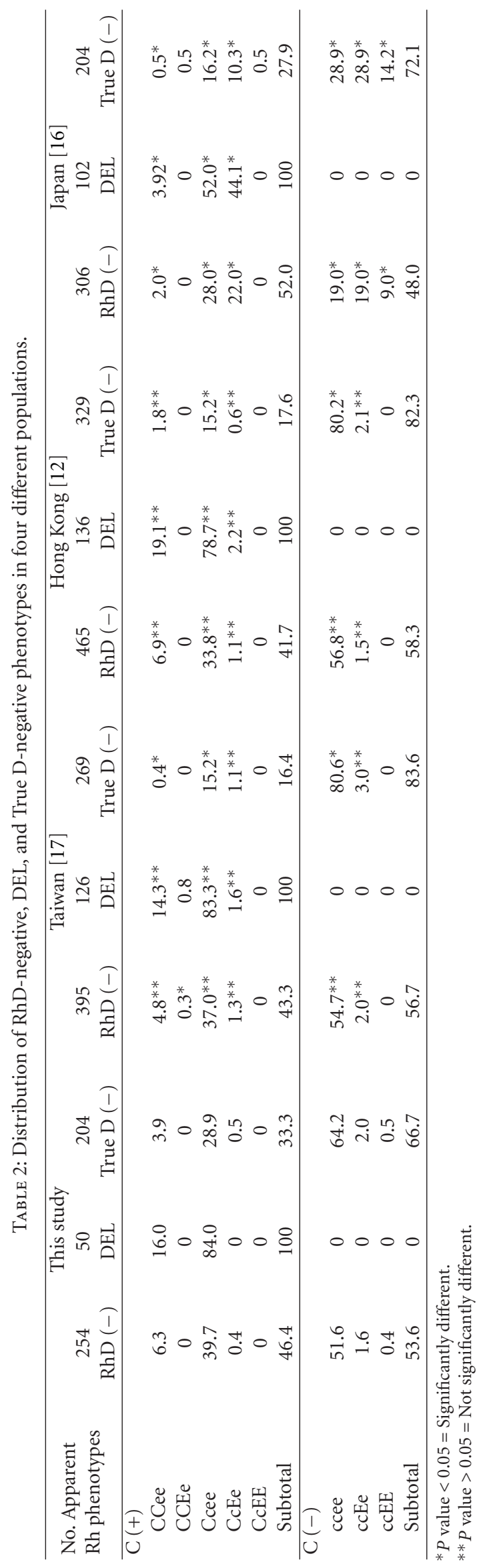


TABLE 3: Results of SSP-PCR for RHD1227A and adsorption/elution in 118 RhC (+) apparent RhD-negative samples.

\begin{tabular}{lcc}
\hline RhC $(+)(N=118)$ & RHD1227A $(+)(N=50)$ & RHD1227A $(-)(N=68)$ \\
\hline Adsorption/elution $(+) N=50$ & 48 & 2 \\
Adsorption/elution $(-) N=68$ & 0 & 68 \\
Sensitivity (\%) & 96 & 100 \\
Specificity (\%) & & \\
\hline
\end{tabular}

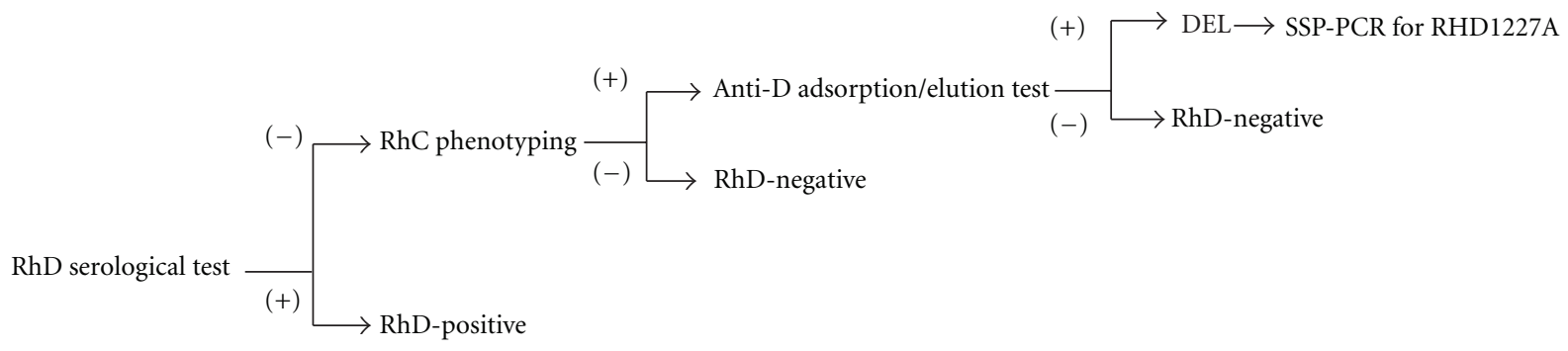

Figure 2: Proposed laboratory protocol for DEL detection.

of the intact RHD gene in DEL samples was related to the $\mathrm{RhC}$ phenotypes with high incidence of $\mathrm{RhC}(+)$ in the apparently RhD-negative persons (46.4\%), and particularly in DEL persons (100\%). All DEL persons with intact $R H D$ gene showed the $\mathrm{Cc}$ and CC phenotype but not the cc phenotype. Almost the same results were demonstrated in the three Asian populations (Taiwan, Hong Kong, and Japan). In addition, by SSP-PCR analysis, the high sensitivity (96\%), and the specificity (100\%) were achieved in DEL person for the detection of RHD 1227A, which is a useful genetic marker for DEL identification. DEL red blood cells can induce alloanti-D immunization in the truly RhD-negative recipients. It is important to distinguish true $\mathrm{RhD}$-negative from DEL phenotype in blood donors. As shown in DEL identification test in blood transfusion laboratory, SSP-PCR for RHD 1227A allele is a reliable and simple method for DEL identification in Thai RhD-negative persons and probably in the other Asian populations. We conclude that $\mathrm{RhC}(+)$ phenotype, anti-D adsorption/elution test and SSP-PCR are benefit for the identification of RHD 1227A polymorphism. Based on these findings, the combined performance test of RhC phenotyping, anti-D adsorption/elution test, and RHD 1227A specific sequence primer-polymerase chain reaction (SSP-PCR) is useful for distinguishing true $\mathrm{RhD}$-negative from DEL phenotype in the Thai RhD-negative blood donor. As shown in Figure 2, phenotype $\mathrm{RhC}(+)$ followed by anti$\mathrm{D}$ adsorption/elution test and RHD 1227A detection can be used as the simple protocol for DEL identification applied in routine laboratory.

\section{Conflict of Interests}

The authors declare that they have no conflict of interests.

\section{Acknowledgments}

This work was supported by Thammasat University research grant for graduate program (2011) and Phramongkutklao
College of Medicine research grant. We thank National Blood Centre, The Thai Red Cross Society, Thailand for support the blood samples.

\section{References}

[1] F. F. Wagner and W. A. Flegel, "RHCE represents the ancestral RH position, while RHD is the duplicated gene," Blood, vol. 99, no. 6, pp. 2272-2273, 2002.

[2] I. Mouro, Y. Colin, B. C. Z. J. P. Cartron, and C. Le Van Kim, "Molecular genetic basis of the human Rhesus blood group system," Nature Genetics, vol. 5, no. 1, pp. 62-65, 1993.

[3] H. Okuda, H. Suganuma, T. Kamesaki et al., "The analysis of nucleotide substitutions, gaps, and recombination events between RHD and RHCE genes through complete sequencing," Biochemical and Biophysical Research Communications, vol. 274, no. 3, pp. 670-683, 2000.

[4] F. F. Wagner and W. A. Flegel, "RHD gene deletion occurred in the Rhesus box,” Blood, vol. 95, no. 12, pp. 3662-3668, 2000.

[5] F. F. Wagner, A. Frohmajer, and W. A. Flegel, "RHD positive haplotypes in D negative Europeans," BMC Genetics, vol. 2, article 10, 2001.

[6] B. K. Singleton, C. A. Green, N. D. Avent et al., "The presence of an RHD pseudogene containing a 37 base pair duplication and a nonsense mutation in africans with the Rh D-negative blood group phenotype," Blood, vol. 95, no. 1, pp. 12-18, 2000.

[7] J. C. Chen, T. M. Lin, Y. L. Chen, Y. H. Wang, Y. T. Jin, and C. T. Yue, "RHD 1227A is an important genetic marker for RhDel individuals," The American Journal of Clinical Pathology, vol. 122, no. 2, pp. 193-198, 2004.

[8] H. Okuda, M. Kawano, S. Iwamoto et al., "The RHD gene is highly detectable in $\mathrm{RhD}$-negative Japanese donors," The Journal of Clinical Investigation, vol. 100, no. 2, pp. 373-379, 1997.

[9] P. Perco, C. P. Shao, W. R. Mayr, S. Panzer, and T. J. Legler, "Testing for the D zygosity with three different methods revealed altered Rhesus boxes and a new weak D type," Transfusion, vol. 43, no. 3, pp. 335-339, 2003.

[10] C. F. Sun, C. S. Chou, N. C. Lai, and W. T. Wang, "RHD gene polymorphisms among RhD-Negative Chinese in Taiwan," Vox Sanguinis, vol. 75, no. 1, pp. 52-57, 1998. 
[11] Y. Okubo, H. Yamaguchi, T. Tomita, and N. Nagao, "A D variant, Del?" Transfusion, vol. 24, no. 6, p. 542, 1984.

[12] K. H. Mak, K. F. Yan, S. S. Cheng, and M. Y. Yuen, "Rh phenotypes of Chinese blood donors in Hong Kong, with special reference to weak D antigens," Transfusion, vol. 33, no. 4, pp. 348-351, 1993.

[13] C. P. Shao, J. H. Maas, Y. Q. Su, M. Köhler, and T. J. Legler, "Molecular background of Rh D-positive, D-negative, Del and weak D phenotypes in Chinese," Vox Sanguinis, vol. 83, no. 2, pp. 156-161, 2002.

[14] J. C. Lan, Q. Chen, D. L. Wu, H. Ding, D. B. Pong, and T. Zhao, "Genetic polymorphism of RhD-negative associated haplotypes in the Chinese," Journal of Human Genetics, vol. 45, no. 4, pp. 224-227, 2000.

[15] Q. Li, L. Y. Ye, Z. H. Guo, M. Qian, and Z. Y. Zhu, "Study on the molecular background of Del phenotype in Chinese population," Chinese Journal of Medical Genetics, vol. 23, no. 5, pp. 486-491, 2006 (Chinese).

[16] Y. Fukumori, Y. Hori, S. Ohnoki et al., "Further analysis of $\mathrm{D}(\mathrm{e} 1)$ (D-elute) using polymerase chain reaction (PCR) with RHD gene-specific primers," Transfusion Medicine, vol. 7, no. 3, pp. 227-231, 1997.

[17] Y. H. Wang, J. C. Chen, K. T. Lin, Y. J. Lee, Y. F. Yang, and T. M. Lin, "Detection of $\mathrm{RhDel}$ in $\mathrm{RhD}$-negative persons in clinical laboratory," Journal of Laboratory and Clinical Medicine, vol. 146, no. 6, pp. 321-325, 2005.

[18] Y. K. Ji, Y. K. Sung, C. A. Kim, S. Y. Gyu, and S. P. Sung, "Molecular characterization of D- Korean persons: development of a diagnostic strategy," Transfusion, vol. 45, no. 3, pp. 345-352, 2005.

[19] T. Wagner, G. F. Körmö, C. Buchta et al., "Anti-D immunization by DEL red blood cells," Transfusion, vol. 45, no. 4, pp. 520-526, 2005.

[20] H. Yasuda, H. Ohto, S. Sakuma, and Y. Ishikawa, "Secondary anti-D immunization by Del red blood cells," Transfusion, vol. 45, no. 10, pp. 1581-1584, 2005.

[21] K. H. Kim, K. E. Kim, K. S. Woo, J. Y. Han, J. M. Kim, and K. U. Park, "Primary anti-D immunization by DEL red blood cells," Korean Journal of Laboratory Medicine, vol. 29, no. 4, pp. 361-365, 2009.

[22] E. Rochna and N. C. Hughes-Jones, "The use of purified 125I-labelled anti-gamma globulin in the determination of the number of D antigen sites on red cells of different phenotypes," Vox Sanguinis, vol. 10, no. 6, pp. 675-686, 1965. 


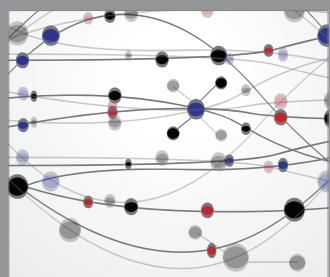

The Scientific World Journal
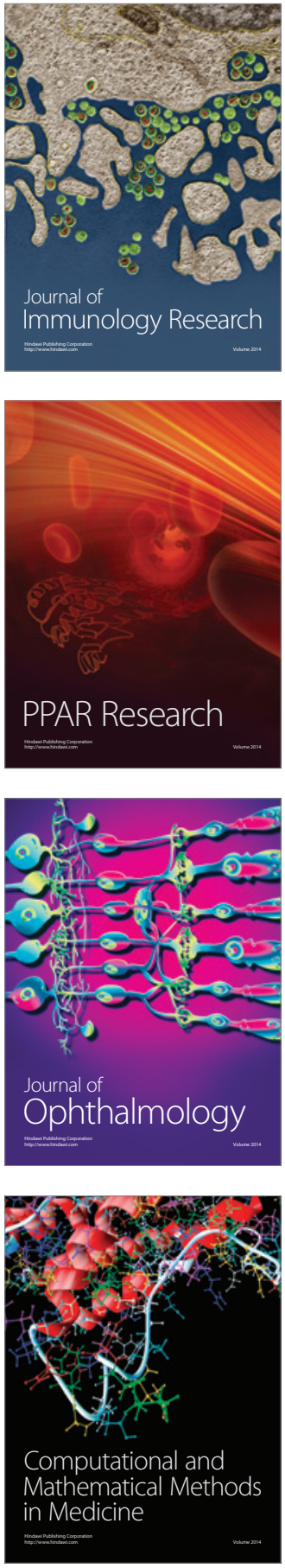

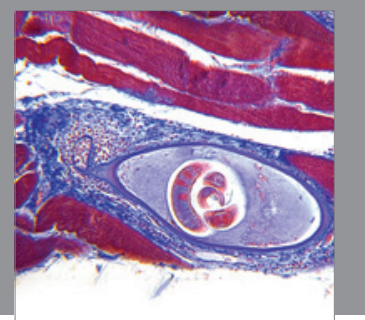

Gastroenterology

Research and Practice
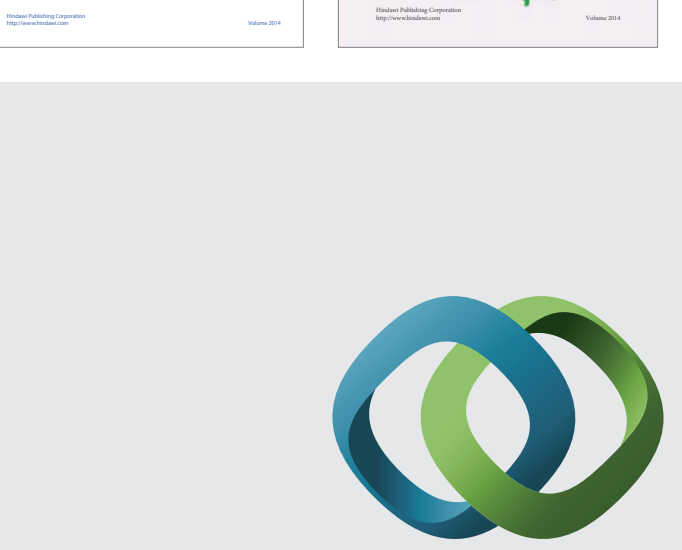

\section{Hindawi}

Submit your manuscripts at

http://www.hindawi.com
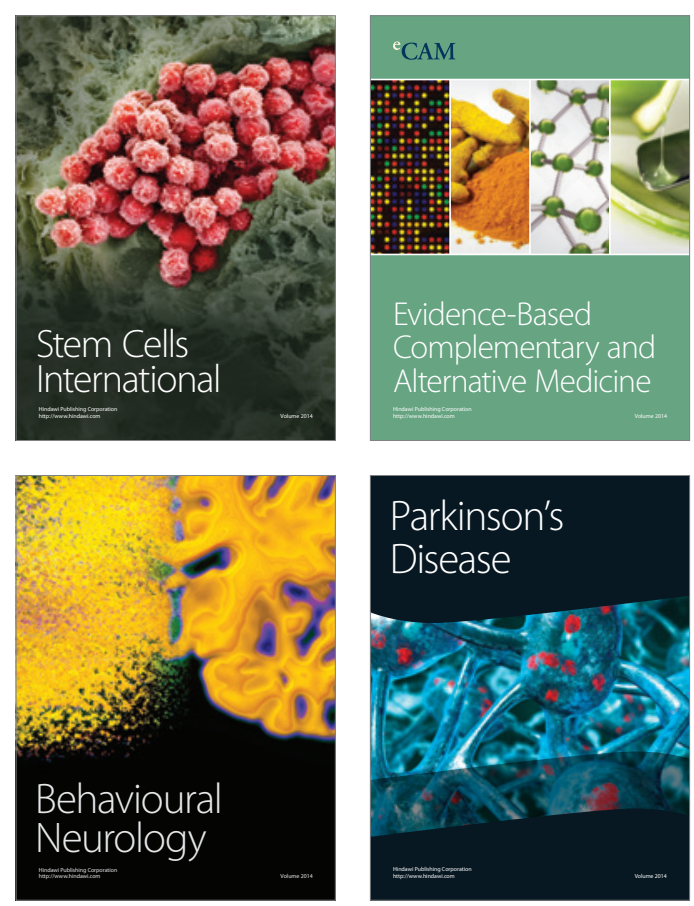

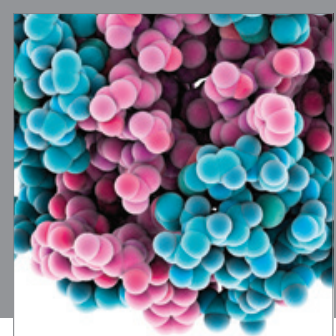

Journal of
Diabetes Research

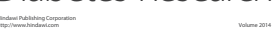

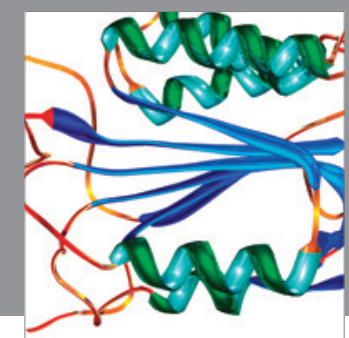

Disease Markers
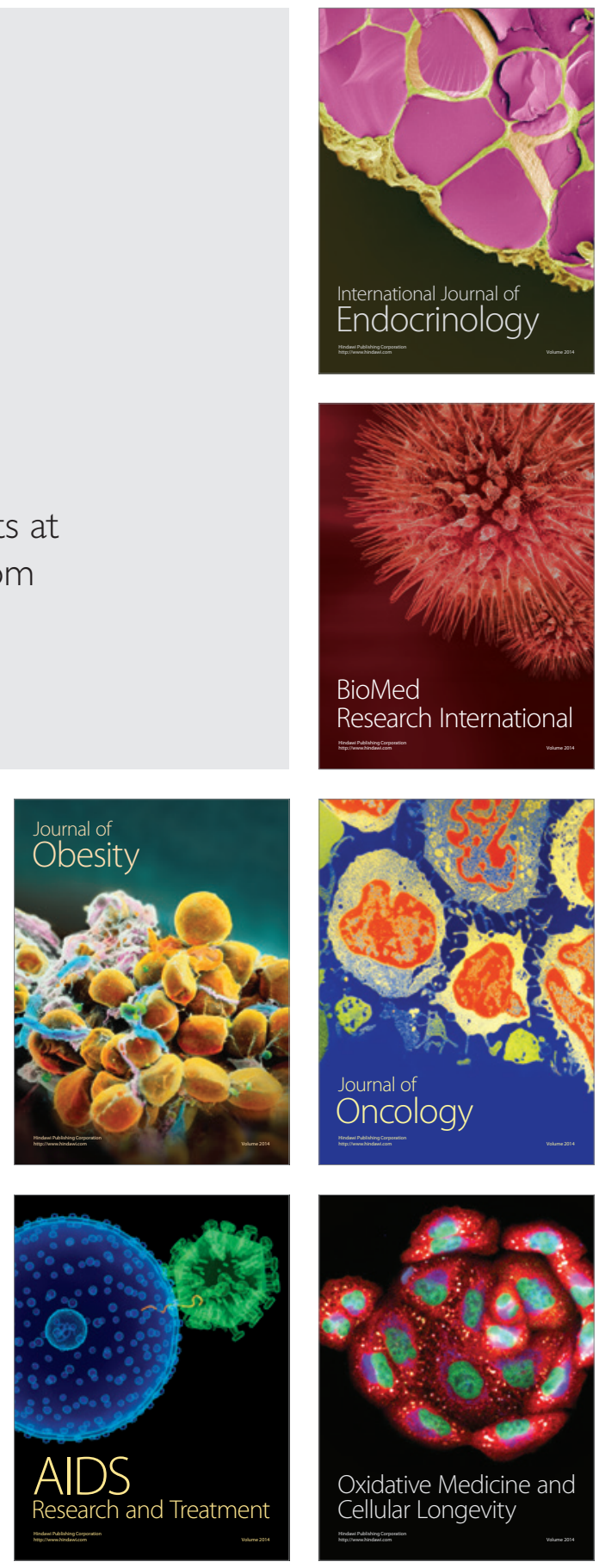